\title{
Study on the Effect of Raindrop Spectrum on Laser Transmission
}

\author{
Yuewei $\mathrm{Wei}^{\mathrm{a}}$, Jun $X \mathrm{u}^{\mathrm{b}}$, Bin $X \mathrm{u}^{\mathrm{c}}$ \\ Xian Research Institute of High Technology, Xi'an 710025, China \\ a245131201@qq.com, bxujun63@sohu.com, '1436135336@qq.com
}

Keywords: atmospheric optics, Laser transmission, raindrop spectrum, attenuation, Mie scattering, transmittance.

\begin{abstract}
The energy of the laser can be greatly reduced, the transmission distance and transmission quality would be affected due to the absorption and scattering of raindrops when transmitting in the rain. Based on Beer-Lambert Law and Mie scattering theory, the impact of raindrop spectrum on 1.06um laser transmission was calculated. Studies have shown that with the increase of rainfall intensity and transmission distance, the laser transmittance decreases. When the rainfall and the transmission distance is consistent, the laser attenuation degree in different raindrop spectrum of rainfall and there are big differences in the change of laser transmittance. This suggests that the raindrop spectrum of rainfall has a big effect on the laser transmission. The study of the effect of raindrop spectrum on laser transmission would help to accurately calculate the laser transmission attenuation characteristic in rain, and improve the quality of the laser transmission in the rain.
\end{abstract}

\section{Introduction}

As a kind of high and new technology, laser is widely used in human life and work. In the use of laser, it is inevitable to meet the rainy day. Rain in the rain will be on the laser scattering and absorption, so that the energy attenuation of the laser, the effect of laser transmission, resulting in the use of limited range of laser ${ }^{[1]}$. The size of rainfall, the length of the transmission distance, and the physical properties of the rainfall itself will affect the transmission of the laser. Raindrop size distribution is one of the important factors of rainfall and physical properties of reaction, which describes the raindrop groups in various radii of the droplet number with the raindrop size distribution, and particles of different sizes of laser scattering and absorption of each are not identical. So it is very necessary to study the influence of the size distribution of raindrops on the laser transmission.

\section{Characteristics of rain}

The cause of the rain is varied, its performance form is also different, is an important part of nature. According to the National Meteorological Bureau of rainfall grade division standard, can be divided into drizzle, light rain, moderate rain, heavy rain and storm rain, their classification as table 1 shows.

Table 1 Classification of rainfall in China Meteorological Administration

\begin{tabular}{cccccc}
\hline Rainfall grade & Drizzle & Light rain & Moderate rain & Heavy rain & Storm rain \\
\hline Rainfall $(\mathrm{mm} / \mathrm{h})$ & $<0.1$ & $0.1 \sim 5$ & $5 \sim 12.5$ & $12.5 \sim 25$ & $25 \sim 100$ \\
\hline
\end{tabular}

\subsection{Shape of raindrops}

The shape of raindrops is mainly determined by the surface tension and the air resistance. And the size of the rain is determined by the surface tension and air resistance which plays a leading role. When the equivalent radius of the raindrops is less than $0.5 \mathrm{~mm}$, the air resistance is much less than the surface tension of the raindrops, and the rain drops are spherical ${ }^{[2]}$. Gradually, the effect of air resistance becomes larger and larger, and the rain drops will become the elliptical shape of the lower flat and the bottom of the depression. When the equivalent radius of the raindrops is more than $6 \mathrm{~mm}$, the 
raindrops will be split from the bottom of the depression, into two or more smaller raindrops, and thus the equivalent radius of the raindrops is generally not more than $6 \mathrm{~mm}$. Therefore, it can be used to calculate the equivalent sphere.

\subsection{Drop velocity}

Due to gravity, buoyancy and air resistance of the role of these three forces, the rain falling in the air to do accelerated movement. Along with the increase of the speed of the rain, the air resistance of the rain is getting bigger and bigger, at the same time, it will be getting smaller and smaller. When these three forces to achieve balance, the acceleration is equal to zero, then the rain will decline at a constant speed, this speed is the end of the drop in the rain ${ }^{[3]}$.

By the drop velocity and other parameters, the rainfall can be calculated, and the distribution of the size distribution of raindrops can also be obtained. On the basis of the experimental data and analysis of other people's data and analysis, the empirical formula of Hale and Querry is obtained:

$$
v(D)=9.65-10.3 e^{-0.6 D}
$$

In the formula, $D$ is the equivalent diameter of raindrops, whose unit is $\mathrm{mm}$.

\section{Raindrops spectrum}

The size distribution of raindrops, which is used to indicate the size distribution of the droplets in the unit volume, is one of the important microscopic characteristics of rainfall. They vary with the elevation of the area, the latitude, the types of the clouds, and the seasons. Although the corresponding rain spectrum measurements have been carried out all over the world, due to the complexity of the rainfall, it is difficult to accurately represent the distribution of the size distribution of the raindrops. Therefore, so far, a large number of models of the rain drops are obtained and used in the research of the corresponding research. In this one, the distribution of Laws-Parsons, Marshall-Palmer distribution, and Weibull distribution of raindrop spectrum model was accepted, and applied in the experimental and theoretical study on the daily ${ }^{[4]}$.

\subsection{Laws-Parsons distribution}

Laws and Parsons have found that the same amount of rainfall in the same area is not the same as the size distribution of the same rainfall in the same area. They will be the same rainfall of the size of the average rainfall data, thus obtained the dispersion of the average size distribution of raindrops, that is, L-P distribution ${ }^{[5]}$. After that, David is fitted to the discrete L-P distribution, and the continuous L-P distribution is obtained:

$$
N(D)=\frac{6 W f(D)}{\pi D^{3}}
$$

In the formula, $f(D)$ is the expression of the volume distribution of raindrops, $N(D)$ is the size distribution function of raindrops, and the distribution of particles with different diameters is changed. Among them,

$$
\begin{aligned}
& f(D)=33.44 R^{-1.28} D^{5.93} e^{-0.538 R^{-0.186} D} \\
& W=\frac{R}{3.6 \times 10^{6} U} \\
& U=\int_{0}^{\infty} f(D) v(D) \mathrm{d} D
\end{aligned}
$$

Among them, $R$ for rainfall, the unit is $\mathrm{mm} / \mathrm{h}$. 


\subsection{Marshall-Palmer distribution}

Through summarizing the advantages and disadvantages of L-P distribution model, and Marshall Palmer in processing their actual measured raindrop data, a new raindrop spectrum model, the exponential distribution model, also known as the M-P distribution ${ }^{[6]}$. Compared to the L-P distribution, the formula of M-P distribution is more concise:

$$
N(D)=N_{0} e^{-\Lambda D}
$$

In the formula, $N_{0}=8000 \mathrm{~m}^{-3} \mathrm{~mm}^{-1}, \Lambda=4.1 R^{-0.21} \mathrm{~mm}^{-1}$.

\subsection{Weibull distribution}

In statistics, Weibull distribution for reliability analysis, which indicates the probability distribution is continuous, the probability density function. Sekine and Lind when they get the raindrops the size of the data analysis, combined with probability theory and statistics, is proposed to describe the raindrop distribution of Weibull distribution:

$$
N(D)=N_{0} \frac{\eta}{\sigma}\left(\frac{D}{\sigma}\right)^{\eta-1} e^{-\left(\frac{D}{\sigma}\right)^{\eta}}
$$

In the formula, usually take $N_{0}=1000 \mathrm{~m}^{-3}, \eta=0.95 R^{0.14}, \sigma=0.26 R^{0.42}$.

\section{Calculation of laser transmission characteristics in the rain}

When the laser is transmitted in the rain, the energy of the particles will be greatly reduced due to the absorption and scattering of the rain particles, so that it can be restricted by the use. Through the beer Lambert law, in Mie scattering theory, analysis of a drop of rain raindrop size distribution spectrum of 1.06um optical propagation effects.

\subsection{Beer-Lambert Law}

By the Beer-Lambert Law, when the light transmission in the rain, fog, haze and other aerosols, the light intensity will be reduced gradually. It applies to monochromatic radiation attenuation can be used to calculate the energy of 1.06um laser propagation in the rain attenuation and the expression:

$$
T=\frac{P_{t}}{P_{0}}=e^{-\beta L}
$$

Where $T$ is the laser transmittance, said transmission light intensity and the incident light intensity ratio, reaction of the laser energy attenuation degree; $P_{\mathrm{t}}$ and $P_{0}$ were transmitted light intensity and the incident light intensity; $L$ for laser transmission distances in the rain; $\beta$ is the extinction coefficient. $N(D)$ can be calculated by the total cross-sectional area $\sigma_{\mathrm{e}}$ and the size distribution function of raindrops ${ }^{[7]}$ :

$$
\beta=\int_{0}^{\infty} \sigma_{\mathrm{e}} N(D) \mathrm{d} D
$$

The total cross-sectional area $\sigma_{\mathrm{e}}$ can be calculated by the geometric area of the extinction efficiency factor $Q_{\mathrm{e}}$ and the spherical particle:

$$
\sigma_{\mathrm{e}}=\frac{\pi D^{2} Q_{\mathrm{e}}}{4}
$$

The extinction efficiency factor $Q_{\mathrm{e}}$ can be obtained by the Mie scattering theory. Mie scattering theory is a classical algorithm which is widely used to deal with the scattering of particles. It is an analytical solution to the interaction between spherical scattering body and electromagnetic wave field. From the shape of the rain, we can know that we can use the rain as the equivalent sphere to deal with. So we can use the Mie scattering theory to calculate the interaction between the raindrops and the laser ${ }^{[8]}$. Its formula is: 


$$
Q_{\mathrm{e}}=\frac{2}{\alpha^{2}} \operatorname{Re} \sum_{m=1}^{\infty}(2 m+1)\left(a_{m}+b_{m}\right)
$$

In the formula, $\alpha$ represents the particle size parameter, $\alpha=2 \pi r / \lambda . a_{\mathrm{m}}, b_{\mathrm{m}}$ is the Mie scattering coefficient, and its formula is:

$$
\begin{aligned}
& a_{m}=\frac{\Psi_{m}^{\prime}(n \alpha) \Psi_{m}(\alpha)-n \Psi_{m}(n \alpha) \Psi_{m}^{\prime}(\alpha)}{\Psi_{m}^{\prime}(n \alpha) Z_{m}(\alpha)-n \Psi_{m}(n \alpha) Z_{m}^{\prime}(\alpha)} \\
& b_{m}=\frac{n \Psi_{m}^{\prime}(n \alpha) \Psi_{m}(\alpha)-\Psi_{m}(n \alpha) \Psi_{m}^{\prime}(\alpha)}{n \Psi_{m}^{\prime}(n \alpha) Z_{m}(\alpha)-\Psi_{m}(n \alpha) Z_{m}^{\prime}(\alpha)} \\
& \Psi_{m}(\rho)=\rho j_{m}(\rho)=\sqrt{\frac{\pi \rho}{2}} J_{m+1 / 2}(\rho) \\
& Z_{m}(\rho)=\rho h_{m}^{(2)}(\rho)=\sqrt{\frac{\pi \rho}{2}} H_{m+1 / 2}(\rho)
\end{aligned}
$$

In the formula, $n$ is the refractive index, and the frequency of the laser light. $J_{\mathrm{m}+1 / 2}(\rho)$ is the Bessel function, $H_{\mathrm{m}+1 / 2}(\rho)$ is Hankel function.

By the above formula, the relationship between the extinction efficiency factor $Q_{\mathrm{e}}$ and the particle size parameter $\alpha$ of the 1.06um laser can be obtained as shown in figure 1 . From the $Q_{\mathrm{e}}$ and the relationship between the curve can be learned that when the $\alpha$ is getting bigger and bigger, the value of

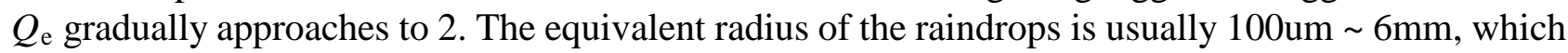
is a large size particle. The value of $\alpha=2 \pi r / \lambda$ that is very large, so it can make $Q_{\mathrm{e}}=2$.

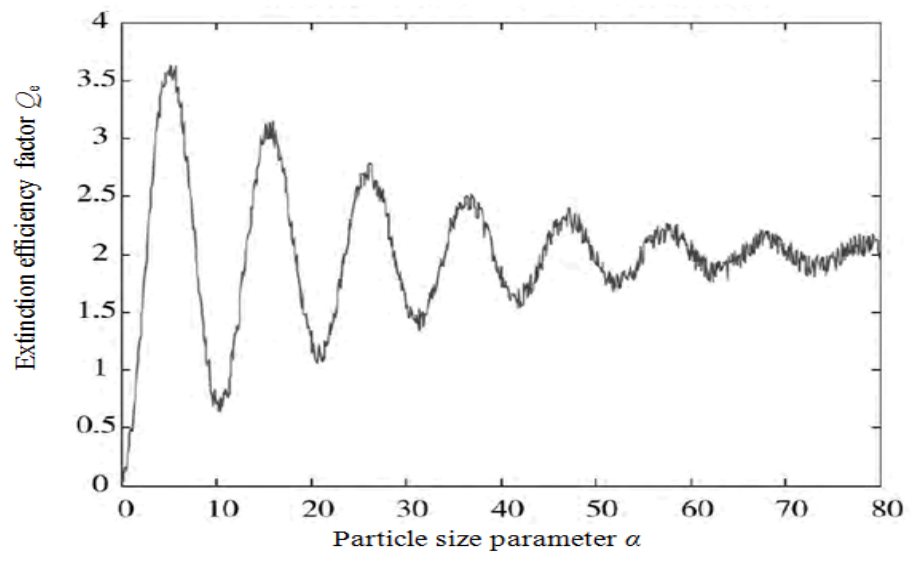

Fig.1 The curve of extinction efficiency factor $Q_{\mathrm{e}}$

\subsection{Calculation of the attenuation of laser in different rain drops}

By formula (9), the variation law of extinction coefficient $\beta$ of 1.06 um laser can be obtained from $0 \mathrm{~mm} / \mathrm{h}$ to $100 \mathrm{~mm} / \mathrm{h}$ under different rainfall patterns. It can be seen from Figure 2 that the extinction coefficient $\beta$ increases with the increase of rainfall, and the increase amplitude of extinction coefficient has obvious difference in different rain drops. Show that laser energy and greatly reduce with increase of rainfall; and under the same rainfall, laser in the M-P distribution of rainfall in the largest extinction coefficient, in the distribution of L-P minimum, the M-P distribution spectral extinction coefficient is about L-P distribution of 2 times. That is to say when the precipitation phase at the same time, laser energy in different raindrop spectrum amplitude attenuation difference. This shows that the precipitation of rain will have an important influence on the laser transmission. 


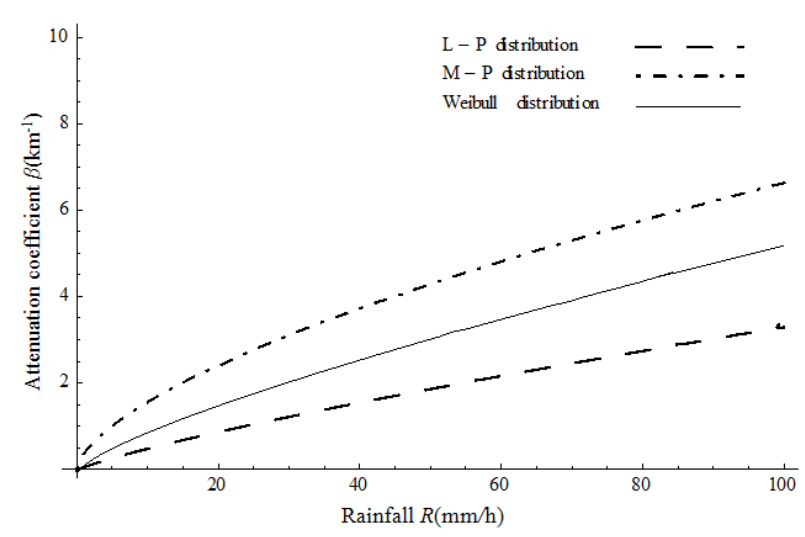

Fig.2 The trend of attenuation coefficient as rainfall changes

Figure 3 respectively show the laser in the transmission distance of $1 \mathrm{~km}$, in the different raindrops spectrum type of rainfall, the rainfall in the $5 \mathrm{~mm} / \mathrm{h}, 20 \mathrm{~mm} / \mathrm{h}, 80 \mathrm{~mm} / \mathrm{h}$, the numerical curve. It can be seen from the figure that the increase of rainfall, the increase of transmission distance, will make the laser transmission rate becomes smaller, and the attenuation of laser transmission rate is obviously related to the characteristics of rain. Transmission distance of $1 \mathrm{~km}$, rainfall is $5 \mathrm{~mm}$, laser L-P in the distribution of rainfall in the transmittance was $75 \%$, in the M-P distribution spectrum transmittance was $36 \%$, in Weibull distribution spectrum through the rate of 53\%. When the transmission distance unchanged, the increase of precipitation is $20 \mathrm{~mm} / \mathrm{h}$. Laser in L-P distribution, M-P distribution and Weibull distribution of rainfall transmittance respectively for $40 \%, 9.5 \%$ and $19 \%$. In the transmission distance for $0.5 \mathrm{~km}$, rainfall is $80 \mathrm{~mm} / \mathrm{h}$ rainstorm, the distribution of laser in L-P, M-P distribution and Weibull distribution of transmittance rapidly declined 25\%, 5.4\% and 10\%. So it can be found that, in the case of the same rainfall and the transmission distance, the distribution of rain drops has a great influence on the laser transmission.

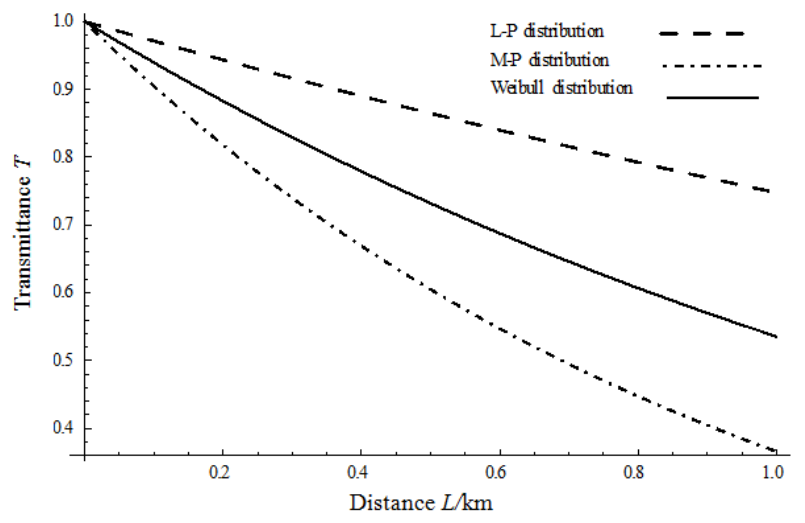

(a) $R=5 \mathrm{~mm} / \mathrm{h}$

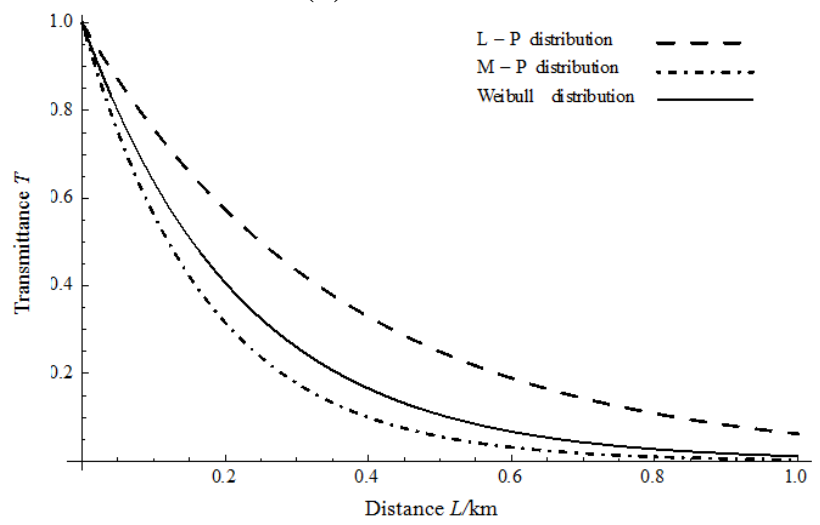

(b) $R=80 \mathrm{~mm} / \mathrm{h}$

Fig.3 The laser transmittance within $1 \mathrm{~km}$ 


\section{Summary}

Through the Beer-Lambert Law, in based on the Mie scattering theory, in different distance and different rainfall. Analysis compared the distribution of laser in L-P, M-P distribution and Weibull distribution of three different raindrop spectra of rainfall in transmission, their extinction coefficient and laser through changes in the rate. The research results show that the 1.06um laser transmission in the rain, proportional to the extinction coefficient and rainfall; laser transmittance and extinction coefficient is inversely proportional to, with the increase of rainfall and the transmission distance and lower; and rainfall raindrops spectral type of laser transmission has great influence, laser in different raindrop spectra in the energy attenuation differences exist. This is mainly due to the different raindrops spectrum, the size distribution of the rain is very different, and different size of raindrops on the scattering and absorption of the laser is not the same. Thus, the extinction coefficient and the laser transmittance of the laser have a great difference in different rain drops.

\section{References}

[1] Hu Zhonghua, Chen Jiabi, Liu Ya. A study of transmission through rain. College Physics, 2007, 26(7): 34-39.

[2] Cheng Jinbo. Study on the analyses and calculate of rain attenuation of Ka-band satellite communication system. Xi’an: Xidian University, 2014

[3] Liu Chenzhong, ZHOU Yunjun, Gu Juan, et al. Characteristics of raindrop size distribution in Chengdu. Journal of Applied Meteorological Science, 2015, 26(1):112-121.

[4] Li Weimin, Ao Faliang, Yu Shengyu. Study of forward scattering effections on laser transformation in raindrop. Photon Technolog, 2006(4): 237-240.

[5] Ke Xizheng, Yang Lihong, Ma Dongdong Transmitted attenuation of laser signal in rain. Infrared and Laser Engineering, 2008, 37(60): 1021-1024.

[6] Li Di, Chen Hui. Study on attenuation characteristic of laser propagation in rain and fog. Electronic Design Engineering, 2011, 19(9): 1-5

[7] Wang Linlin, Duan Jin, Fu Jun, et al. Analysis on the impact of rainfall on laser transmission distance. Applied Laser. 2015,35(1): 99-103.

[8] Liu Jianbin, Wu Jian. Study of scattering property of spherical particle in atmoshere. Applied Optics. 2005, 26(2): 31-33 